\title{
Spin Zero Quantum Relativistic Particles in Einstein Universe
}

\author{
M. R. Setare ${ }^{1}$, Khaled Saaidi ${ }^{2}$ \\ Institute for Studies in Theoretical Physics and Mathematics, P.O.Box, 19395-5531, Tehran, Iran \\ Department of Science, University of Kurdistan, Pasdaran Ave., Sanandaj, Iran
}

\begin{abstract}
In this letter we have considered the eigenvalues and eigenfunctions of relativistic massless scalar particle which conformally coupled to the background of Einstein universe. We found the eigenvalues and eigenfunctions exactly.
\end{abstract}

\footnotetext{
${ }^{1}$ E-mail: rzakord@ipm.ir

${ }^{2}$ E-mail: KSaaidi@ipm.ir
} 


\section{Introduction}

The one of the most interesting problem in theoretical physics is the connection between the quantum mechanics and gravity. Since the birth of quantum mechanics there has been many work in this topic [1]-[11]. Chandrasekhar, studied the Dirac equation in a Kerr space-time background. He separated the Dirac equation into radial and angular parts[2]. The Page extended this work to the Dirac equation for an electron around a Kerr-Newman back hole back ground, in this case also the Dirac equation is separated into decoupled ordinary differential equation[3]. The separation of variables for the massive complex Dirac equation in the gravitational back ground of the Dyon black hole has been done in [5]. Also, in [6] the radial part of Dirac equation in a Schwarzschild geometry has been solved by using WKB approximation method. Moreover above theoretical works several experiments have been performed to test theoretical predications, for example the authors of [12] have been used a neutron interferometer to observe the quantum mechanical phase shift of neutrons caused by their interaction with the gravitational field of Earth. Furthermore, Nesvizhevsky et al [13] have been measured the quantum energy levels of neutron in the Earth's gravitational field. In this letter we shall consider the eigenvalues and eigenfunctions of relativistic massless scalar particle which conformally coupled to the background of Einstein universe. We will find the eigenvalues and eigenfunctions exactly.

\section{Massless conformally coupled spin zero quantum relativistic particles}

The simplest cases in which the analysis of quantum behavior of particles can be done is the massless spin zero particles in the static spacetime. Here we consider the Einstein universe, which scalar particle conformally coupled it. The line element for, this space is given by

$$
d s^{2}=d t^{2}-a^{2} d \chi^{2}+\sin (\chi)^{2}\left(d \theta^{2}+\sin ^{2} \theta d \varphi^{2}\right),
$$

in this case the scalar curvature is as following

$$
R=\frac{6}{a^{2}}
$$

The equation of motion for massless scalar particles is given by

$$
(\square+\xi R) u(x)=0,
$$

where $\square$ is given by

$$
\square=(-g)^{\frac{1}{2}} \partial_{\mu}\left[(-g)^{\frac{1}{2}} g^{\mu \nu} \partial_{\nu}\right]
$$

The modes $u(x)$ are separated as

$$
u_{k}(x)=\frac{1}{a} y_{k}(x) \chi_{k}(t)
$$

with $x=(\chi, \theta, \varphi)$ and $y_{k}(x)$ is a solution of

$$
\Delta^{3} y_{k}(x)=-\left(k^{2}-1\right) y_{k}(x),
$$


with this separation $\chi_{k}$ satisfies following equation

$$
\frac{d^{2} \chi_{k}}{d \eta^{2}}+\left[k^{2}+c(\eta)[\xi-\xi(4)] R(\eta)\right] \chi_{k}=0,
$$

with $\xi(4)=\frac{1}{6}$, also $c(\eta)=a^{2}(t)$, which the conformal time parameter $\eta$ given by

$$
\eta=\int^{t} a^{-1}\left(t^{\prime}\right) d t^{\prime}
$$

Using the scalar curvature $\mathrm{Eq}(2)$, the normalized solution of $\mathrm{Eq}(1)$ can be written as

$$
\chi_{k}(\eta)=\left(2 \omega_{k}\right)^{-1 / 2} e^{-i \omega_{k} \eta}
$$

where

$$
\omega_{k}^{2}=k^{2}+(6 \xi-1)
$$

For conformally coupled case $\xi=\frac{1}{6}$ we have

$$
\omega_{k}^{2}=k^{2}, \quad k=1,2, \ldots
$$

Which $\omega_{k}=k$ are eigenvalues of massless scalar particles in our interest background. Therefore the eigenvalues are equally spacing. The eigenfunctions $y_{k}$ of the three-dimensional Laplacian are $[15,16]$

$$
y_{k}(x)=\Pi_{K j}(\chi) Y_{j}^{m}(\theta, \varphi), \quad K=(k, j, M)
$$

where

$$
M=-j,-j+1, \ldots, j ; \quad j=0,1, \ldots, k-1 \quad k=1,2, \ldots
$$

The $Y_{j}^{M}$ are spherical harmonics. The functions $\Pi_{K j}^{(+)}(\chi)$ are defined by [14]

$$
\Pi_{K j}^{(+)}(\chi)=\left\{\frac{1}{2} \pi(i k)^{2}\left[(i k)^{2}+1^{2}\right] \ldots\left[(i k)^{2}+j^{2}\right]\right\}^{\frac{-1}{2}} \sinh ^{j}(i \chi)\left(\frac{d}{d \cosh (i \chi)}\right)^{1+j} \cos (k \chi) .
$$

\section{References}

[1] L. D. Landau and E. M. Lifshitz; Quantum Mechanics-Non-relativistic Theory (Pergamon Press, 1977).

[2] S. Chandrasekhar; Proc. R. Soc. Lond. A349 (1976) 571.

[3] D. N. Page; Phys. Rev. D14 (1976) 1509.

[4] S. K. Chakrabarti, Proc. R. Soc. Lond. A391 (1984) 27.

[5] I. Semiz; Phys. Rev. D46 (1992) 5414.

[6] B. Mukhopadhyay \& S. K. Chakrabarti; Class. Quant. Grav. 16 (1999) 3165.

[7] D. V. Ahluwalia \& C. Burgard; Gen. Rel. Garv. 28 (1996) 1161. 
[8] C. Y. Cardall \& G. M. Fuller; Phys. Rev. D55 (1997) 7960.

[9] M. Alimohammadi \& A. Shariati; Int. J. Mod. Phys. A15 (2000) 4099.

[10] M. Khorrami, M. Alimohammadi, A. Shariati, Annals Phys. 304, 91, (2003).

[11] M. Alimohammadi, B. Vakili, gr-qc/0306126.

[12] R. Colella, A. W. Overhauser, \& S. A. Werner; Phys. Rev. Lett. 34 (1975) 1472.

[13] V. V. Nesvizhevsky et al.; Nature 415 (2002) 297.

[14] E. M. Lifshitz, I. M. khalatnikov, Adv. Phys. 12 ,185,(1963)

[15] L. Parker, S.A.Fulling, Phys.Rev. D9, 341, (1974).

[16] N. D. Birrell, P. C. W. Davies, Quantum Fields in Curved Space, Cambridge University Press, 1982. 